\title{
Skip lymph node metastasis in gastric cancer: is it skipping or skipped?
}

\author{
Yoon Young Choi $\cdot$ Ji Yeong An $\cdot$ Ali Guner $\cdot$ Dae Ryong Kang $\cdot$ \\ In Cho • In Gyu Kwon · Hyun Beak Shin · Woo Jin Hyung • Sung Hoon Noh
}

Received: 8 October 2014/Accepted: 25 January 2015/Published online: 24 February 2015

(c) The International Gastric Cancer Association and The Japanese Gastric Cancer Association 2015

\begin{abstract}
Background Skip metastasis is the presence of a metastatic lymph node (LN) in an extraperigastric (EP) area without perigastric (PG) involvement. The mechanism and prognosis of skip metastasis are still unknown. The purpose of this study was to scrutinize the clinical significance of skip metastasis in gastric cancer.

Methods Data were reviewed from 6,025 patients who had undergone gastrectomy for primary gastric cancer. Patients were categorized as a PG-only group when the metastatic LNs were limited to only the PG area, as a PG + EP group if metastatic LNs extended to both the PG area and the EP area,
\end{abstract}

Y. Y. Choi and J. Y. An contributed equally to this work.

Y. Y. Choi · J. Y. An · A. Guner - I. Cho ·

I. G. Kwon · H. B. Shin · W. J. Hyung · S. H. Noh ( $ه)$

Department of Surgery, Yonsei University Health System,

Yonsei University College of Medicine, 50 Yonsei-Ro,

Seodaemun-gu, Seoul 120-752, Republic of Korea

e-mail: jar319@yuhs.ac; sunghoonn@yuhs.ac

D. R. Kang

Department of Medical Humanities \& Social Medicine, Office of

Biostatistics, Ajou University School of Medicine, Seoul,

Republic of Korea

I. Cho

Department of Surgery, Catholic Kwandong University College of Medicine, International St. Mary's Hospital, Incheon,

Republic of Korea

I. G. Kwon

Department of Surgery, Keimyung University School of Medicine, Daegu, Republic of Korea

S. H. Noh

Brain Korea 21 PLUS Project for Medical Science, Seoul, Republic of Korea and as a skip group if metastatic LNs were in the EP area but there were no metastatic LNs in the PG area.

Results After we had performed matching, the prognosis of the skip group was worse than that of the PG-only group (adjusted hazard ratio 1.69, $95 \%$ confidence interval 1.13-2.54) and was similar to that of the PG + EP group (adjusted hazard ratio: $1.54,95 \%$ confidence interval 0.92-2.59). The number of retrieved LNs was less in the skip group than in the other groups, especially from the PG area $(p<0.001)$.

Conclusions The prognosis of the skip group was worse than that of the PG-only group and was similar to that of the PG + EP group when the tumor stage was considered. It is difficult to conclude whether skip metastasis is real skipping of cancer cells or a result of inadequate LN sampling. Further evaluation of LNs in the PG area of the skip group could provide more clues for the mechanism of skip metastasis.

Keywords Gastric cancer - Skip metastasis - Prognosis

\section{Introduction}

The lymphatic system is one of the main routes for cancer spread, and lymph node (LN) metastasis is an important prognostic factor in gastric cancer [2, 3]. In theory, cancer cells spread stepwise, meaning that $\mathrm{LN}$ involvement in cancer should first be from the area closest to the tumor. However, sometimes metastatic LNs can be detected far from the original tumor without being detected in the peritumoral area, which has been called skip metastasis. This unusual pattern of $\mathrm{LN}$ metastasis has been reported in various cancers, such as breast, colon, and lung cancer [3-5]. Several studies have also reported on skip metastasis in gastric cancer [7-9], in which 
the following factors could play a role: (1) occult metastasis or micrometastasis which was missed during routine examination of the perigastric (PG) area [10]; (2) direct lymphatic flow from the tumor to LNs in the extraperigastric (EP) area, bypassing the PG area [11-13; and (3) free cancer cells passing through PG LNs because of an unfit microenvironment for settling [14]. Despite previous reports, the prognosis and mechanism of skip metastasis in gastric cancer are not congruent because the number of cases in each study was limited. We reviewed our large volume of data and the previous literature to scrutinize the clinical significance and mechanism of skip metastasis.

\section{Methods}

Included participants

We reviewed the data of 9,350 patients who had undergone gastrectomy for primary gastric cancer at Yonsei University Hospital from January 2000 to December 2010. The strategy for the extent of LN dissection was as follows: D1+ or D2 lymphadenectomy was performed for clinically early gastric cancer, and D2 or greater lymphadenectomy was performed for clinically advanced gastric cancer. All of the data were prospectively collected and retrospectively reviewed. The survival of each patient was verified on the basis of hospital records, telephone calls, and the database of the Korea National Statistical Office. This study was approved by the Institutional Review Board of our hospital (4-2013-0854).

The criteria of the present study were the same as our previous protocol (NCT01961791). Patients who had undergone minimally invasive surgery were excluded ( $n=1,480)$, as were patients with distant metastasis ( $n=303$ ). The final outcomes of 6,025 patients were analyzed after we had also excluded patients if the LN locations were not divided ( $n=986$ ), if the metastatic LN location was unknown $(n=474)$, and if patients had received preoperative chemotherapy $(n=82)$.

The definition of the skip group and other groups

The LNs around the stomach were categorized in terms of PG area (stations 1, 2, 3, 4, 5, and 6 according to the Japanese gastric cancer classification) [15] and EP area (stations 7, 8, 9, 10, 11, 12, etc.). A skip metastasis was defined as being present when there was a metastatic LN in the EP area but no metastatic LN in the PG area, and these patients were categorized as the skip group. Patients were categorized as a PG-only group if metastatic LNs were localized in only the PG area and not the EP area. Patients were categorized as a PG + EP group if the gastric cancer involved LNs in both the PG area and the EP area.
Propensity scoring match and statistical analysis

Propensity score analyses were done to handle selection bias and to estimate the prognosis of the skip group compared with the other groups [15]. Propensity scores were obtained by logistic regression analysis with variables concerning patients (age and sex), gastric cancer (pT stage, the number of metastatic LNs, sizes, Lauren classification, and histologic type), and operation (extent of gastrectomy, operation time, the number of LNs retrieved, and length of hospital stay in days). The model discrimination was assessed by the $c$ statistic, and calibration was assessed by the Hosmer-Lemeshow test.

Categorical variables were compared by the chi square test or Fisher's exact test, and continuous variables were compared by the $t$ test. Overall survival (OS), which was defined as death from any cause, and disease-free survival (DFS), which was defined as recurrence or death from any cause, whichever came first after the operation, were used to assess the prognosis of each group, and the Kaplan-Meier method and the log-rank test were used to compare the prognoses. The Cox proportional hazards model was used to estimate hazard ratios (HRs), and the variables that can affect the prognosis of gastric cancer (such as pT stage, the number of metastatic LNs, age, and sex) were adjusted to estimate the adjusted HRs after the match. Every comparison was limited to only the skip group and the PG-only group, and to the skip group and the $\mathrm{PG}+\mathrm{EP}$ group (in other words, the PG-only and $\mathrm{PG}+\mathrm{EP}$ groups were not compared). The HR is given with its $95 \%$ confidence interval (CI). SPSS Statistics version 20.0 (IBM, Armonk, NY, USA) was used for all statistical analyses, and a two-sided $p$ value of less than 0.05 was considered statistically significant.

Review of the literature

To review the previously published literature, we searched PubMed, EMBASE, and the Cochrane Central Register of Controlled Trials as of October 4, 2013, with the following related keywords: gastric cancer, skip metastasis, and jump metastasis. Only studies written in English were included, and bibliographies of the related articles were manually searched to find additional studies.

\section{Results}

Incidence of skip metastasis

Among the final cohort of patients enrolled in the present study, 2,231 (37.0\%) had metastatic LNs. Specifically, 1,137 of 2,231 patients $(51.0 \%)$ had metastatic LNs around the stomach only (PG area), and 988 patients 
(44.3\%) had metastatic LNs in both the PG area and the EP area. The incidence of skip metastasis was $4.8 \%$ (106 of 2,231 patients) among the patients with metastatic LNs, and $1.8 \%$ among the overall gastric cancer population.

\section{Baseline characteristics of each group}

The baseline characteristics of the skip, PG-only, and PG + EP groups are shown in Table 1. The age, sex, operation time, and hospital length of stay of the skip group were similar to those of the PG-only and PG + EP groups. On the other hand, the pT and $\mathrm{pN}$ stages of the skip group were lower than those of the PG-only and PG + EP groups, and the average tumor size of the skip group was smaller than that of the PG-only and PG + EP groups. Intestinal-type and undifferentiated-type gastric cancer were more frequent in the skip group than in the $\mathrm{PG}+\mathrm{EP}$ group ( $p=0.034$ and $p=0.002$, respectively). In addition, the number of LNs retrieved was less than that in either the PG-only group or the PG + EP group ( $p=0.049$ and $p<0.001$, respectively).

\section{Propensity scoring match}

A 1:4 propensity scoring match was performed to compare the prognosis of the skip group with that of the PG-only group. The $p$ value of the Hosmer-Lemeshow test was 0.927 , and that of the $c$ statistic was 0.719. After we had performed the matching, the baseline characteristics of the skip and PG-only groups were similar for all variables (Table 1). However, a proper match was difficult between the skip and PG + EP groups because the number of metastatic LNs was too different. Therefore, we performed a 1:1 propensity scoring match after excluding 358 patients who were classified as having $\mathrm{pN} 3 \mathrm{~b}$ stage gastric cancer in the PG + EP group as there were no patients who were classified as having pN3b stage gastric cancer (the number of metastatic LNs was more than 15) in the skip group. The $p$ value of the Hosmer-Lemeshow test was below 0.001, and that of the $c$ statistic was 0.968 . After we had performed the matching, the number of metastatic LNs was still higher in the $\mathrm{PG}+\mathrm{EP}$ group than in the skip group $(p<0.001)$.

The number of LNs retrieved in each group

Table 2 shows the number of LNs retrieved in each subgroup. Overall, the number of LNs retrieved in the skip group was less than that retrieved in the PG-only group or the PG + EP group. In cases of distal gastrectomy, the number of LNs retrieved in the skip group was still less than that retrieved in either the PG-only group or the $\mathrm{PG}+\mathrm{EP}$ group. In cases of total gastrectomy, the mean number of LNs retrieved was least in the skip group (48.3 in the skip group, 50.1 in the PG-only group, and 53.1 in the PG + EP group), but this difference was not statistically significant. In the analysis of the number of LNs retrieved from each LN location (PG or EP area), there was a significant difference in the number of LNs retrieved from the PG area among the three groups (21.6 in the skip group, 27.5 in the PG-only group, and 28.1 in the PG + EP group). However, the number of LNs retrieved in the EP area was similar among the three groups.

The location of skip metastasis and the number of involved stations

The commonest location of skip metastasis was station 7 around the left gastric artery (67 of 106 patients, $63.2 \%$ ), and the skip metastasis involved only one station in 83 patients $(79.2 \%$ ) (Table 3). Station 8 (around the common hepatic artery) and station 9 (around the celiac axis) were the next commonest locations of skip metastasis (33.0 and $30.2 \%$, respectively). There was one patient who had nine metastatic LNs with five involved stations, of which all were located in only the EP area. The total number of LNs retrieved from the 106 patients with skip metastasis was 1,787, and the number of metastatic LNs was 212.

Prognosis of the skip group compared with the PG-only group and the PG + EP group

The prognosis of the skip group was similar to that of the PG-only group before matching (log-rank test, $p=0.472$ for OS and $p=0.429$ for DFS) and better than that of the $\mathrm{PG}+\mathrm{EP}$ group (log-rank test, $p<0.001$ for OS and $p<0.001$ for DFS; Figs. 1a, 2a) for overall stage. In a subgroup analysis, the prognosis of the skip group was likely to be worse than that of the PG-only group (log-rank test, $p=0.05$ for OS and $p=0.058$ for DFS; Figs. 1b, 2b) for stage I disease. For stage II disease, there was no difference in the prognosis between the skip group and the PG-only or PG + EP groups (Figs. 1c, 2c). For stage III disease, the prognosis of the skip group seemed to be better than that of the PG + EP group (log-rank test, $p=0.043$ for OS and $p=0.077$ for DFS; Figs. 1d, 2d).

The results after matching showed that the prognosis of the skip group was worse than that of the PG-only group (log-rank test, $p=0.025$ for OS and $p=0.016$ for DFS; Fig. 3a, c). The HR adjusted for pT stage, the number of metastatic LNs, age, and sex after matching was 1.69 (95\% CI 1.13-2.54, $p=0.011)$ for OS and $1.62(95 \% \mathrm{CI}$ $1.11-2.36, p=0.012$ ) for DFS. However, the prognosis of the skip group was similar to that of the $\mathrm{PG}+\mathrm{EP}$ group after matching (log-rank test, $p=0.967$ for OS and $p=0.753$ for DFS; Fig. $3 \mathrm{~b}$, d). The adjusted HR of the 


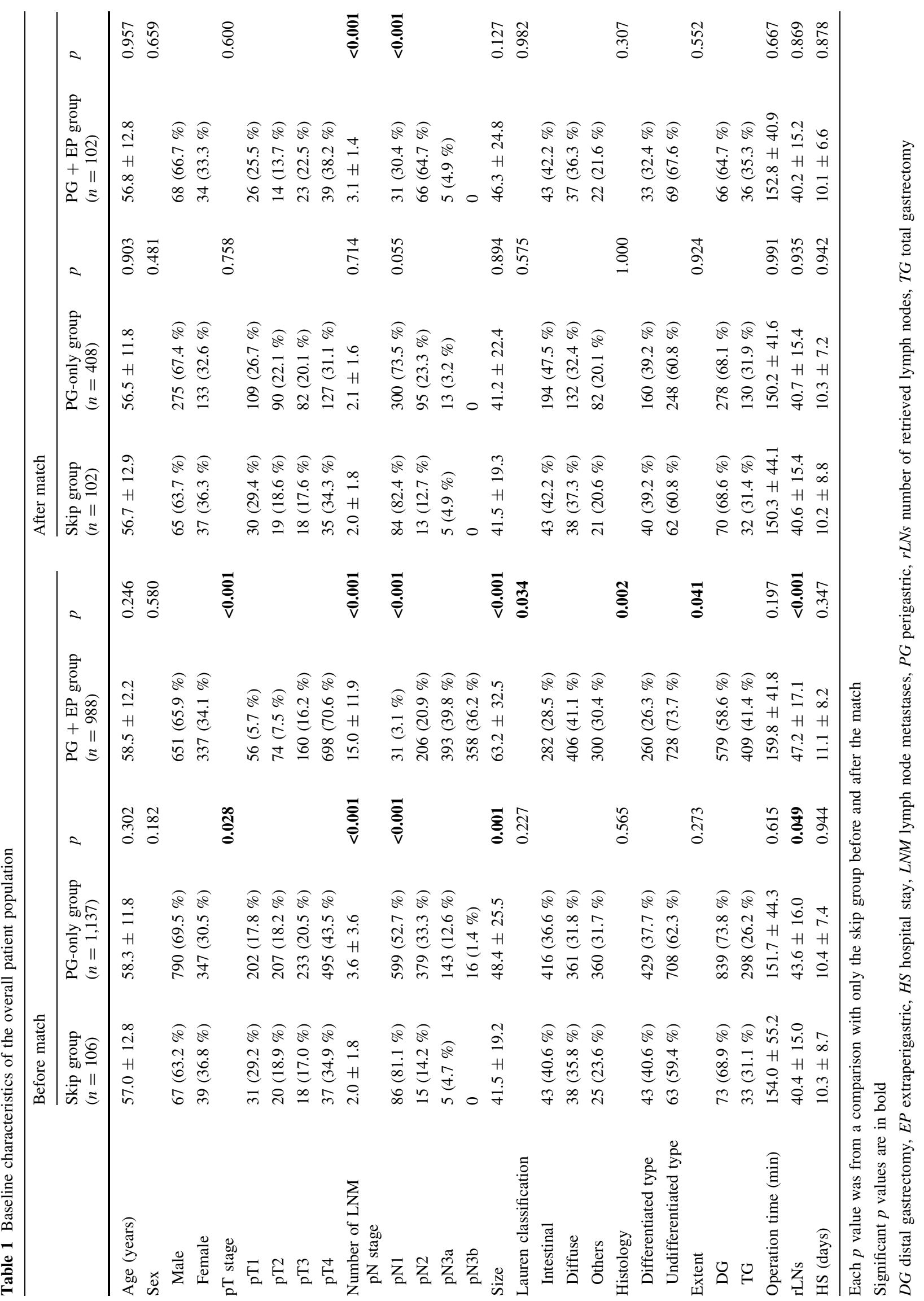


Table 2 The number of retrieved lymph nodes $(L N s)$ in each group

$D G$ distal gastrectomy, $E P$ extraperigastric, $P G$ perigastric, $T G$ total gastrectomy $* p<0.05, * * p<0.001$

\begin{tabular}{llll}
\hline & Skip group $(n=106)$ & PG-only group $(n=1,137)$ & PG + EP group $(n=988)$ \\
\hline $\begin{array}{l}\text { Total } \\
\text { Extent }\end{array}$ & $40.4 \pm 15.0$ & $43.6 \pm 16.0^{*}$ & $47.2 \pm 17.1^{* *}$ \\
DG & $37.1 \pm 11.9$ & $41.3 \pm 14.5^{*}$ & \\
TG & $48.3 \pm 18.4$ & $50.1 \pm 18.0$ & $43.1 \pm 14.4^{*}$ \\
Location of LNs & & $53.1 \pm 18.8$ \\
PG area & $21.6 \pm 10.5$ & $27.5 \pm 12.3^{* *}$ & $28.1 \pm 13.9^{* *}$ \\
EP area & $18.8 \pm 8.7$ & $16.1 \pm 8.8^{*}$ & $19.1 \pm 8.9$ \\
\hline
\end{tabular}

Table 3 The location of skip metastasis and the number of involved stations

\begin{tabular}{lllr}
\hline & $\begin{array}{l}\text { Number of } \\
\text { patients } \\
(n=106)\end{array}$ & $\begin{array}{l}\text { Number of } \\
\text { metastatic } \\
\text { LNs }\end{array}$ & $\begin{array}{r}\text { Ratio of metastatic } \\
\text { LNs/retrieved LNs }\end{array}$ \\
\hline Location of skip LNs & & \\
Station 7 & $67(63.2 \%)$ & 72 & $14.5 \pm 24.6 \%$ \\
Station 8a & $35(33.0 \%)$ & 66 & $17.0 \pm 28.3 \%$ \\
Station 9 & $32(30.2 \%)$ & 38 & $10.8 \pm 16.7 \%$ \\
Station 10 & $5(4.7 \%)$ & 6 & $15.1 \pm 30.2 \%$ \\
Station 11 & $13(12.3 \%)$ & 20 & $9.3 \pm 19.8 \%$ \\
Station 12a & $6(5.7 \%)$ & 7 & $0.5 \pm 20.2 \%$ \\
Stations 13 & $2(1.9 \%)$ & 3 & \\
and 14 & & & \\
Number of & & & \\
involved & & & \\
stations & & & \\
1 & $84(79.2 \%)$ & & \\
2 & $19(17.9 \%)$ & & \\
3 & $2(1.9 \%)$ & & \\
4 & 0 & & \\
5 & 1 & & \\
\hline
\end{tabular}

$L N$ lymph node

a The status of the LNs of this patient was as follows: station $1(0 / 8)$, station $4(0 / 5)$, station $5(0 / 1)$, station $6(0 / 7)$, station $7(4 / 9)$, station $8(2 / 3)$, station $9(0 / 5)$, station $10(1 / 1)$, station $11(1 / 2)$, station 12 (1/1), station 3 (soft tissue free of carcinoma)

skip group compared with the $\mathrm{PG}+\mathrm{EP}$ group after matching was 1.54 (95 \% CI 0.92-2.59, $p=0.102)$ for OS and 1.41 (95\% CI $0.87-2.30, p=0.164)$ for DFS, and did not show statistical significance.

Results of previous studies

A total of 15 studies [6-8, 10, 16-24] were identified by our search strategy with full text review. The populations of these studies ranged from single $\mathrm{LN}$ metastasis cases to whole gastric cancer cohorts because some of the studies focused only on sentinel LN mapping. In the studies, the incidence of skip metastasis among the entire gastric cancer population ranged from 0 to $11.8 \%$, and from 2.4 to $60 \%$ among gastric cancer patients with $\mathrm{LN}$ metastasis. There were only three studies which compared the prognosis of skip metastasis in gastric cancer [9, 11,23], but the control groups for skip metastasis were different between the studies. Two studies reported that there was no statistical difference in prognosis between the skip metastasis group and the other groups $[9,11]$. One study reported that the prognosis of skip metastasis was better than that of gastric cancer with LN metastasis at the N2 level, or when the number of metastatic LNs was between 7 and 15 (pN2 stage according to the 6th edition of the Union for International Cancer Control TNM classification), but was similar to that of gastric cancer with LN metastasis at the N1 level or when the number of metastatic LNs was between one and six (pN1 stage according to the 6th edition of the Union for International Cancer Control TNM classification) [23]. Only one study reported the number of LNs retrieved with skip metastasis, and this was less for those with skip metastasis than for those without skip metastasis [9].

\section{Discussion}

Without any matching or adjustment, our results initially demonstrate that the prognosis of patients in the skip group is similar to that of patients in the PG-only group, but is better than that of patients in the PG + EP group. These findings are consistent with those of previous reports $[9,11$, 23]. However, if we consider the different baseline characteristics and particularly the TNM stage, the prognosis of patients in the skip group is worse than that of patients in the PG-only group but is similar to that of patients in the PG + EP group. These findings are similar to those in a previous report [26] that found that the anatomical extent of metastatic LNs is still important in the prognosis of gastric cancer. In other words, even though the pT stage and the number of metastatic LNs are similar, the prognosis is worse if the metastatic LNs extend to the EP area than if they are limited to only the PG area. It is also possible that there are missed metastatic LNs or micrometastases in the PG area. Natsugoe et al. [27] reported that reexamination with additional sections of LNs identified more metastatic 

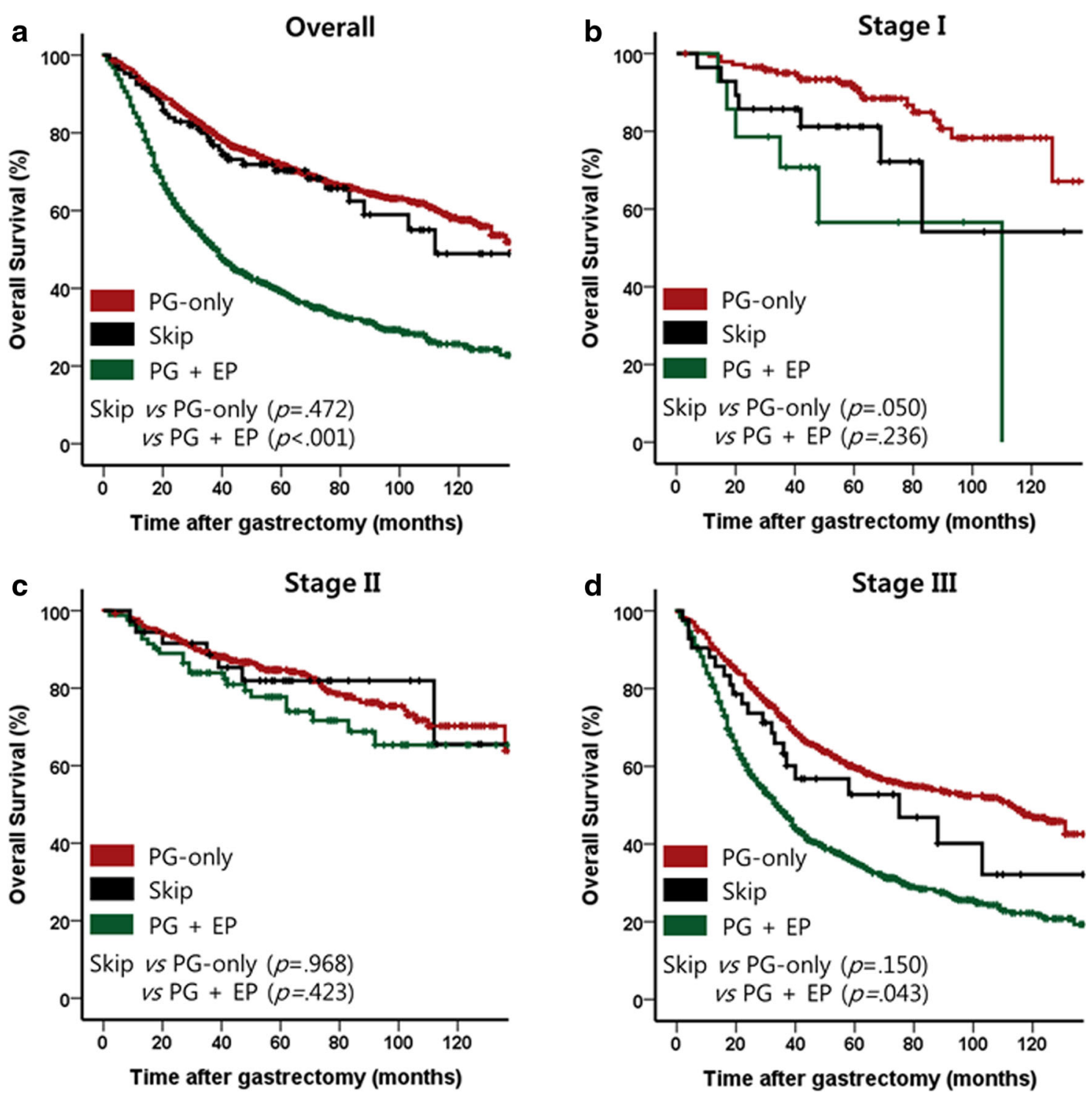

Fig. 1 Overall survival of each status of lymph node metastasis with Kaplan-Meier curves before matching: a for overall stage, b for stage I disease, $\mathbf{c}$ for stage II disease, and $\mathbf{d}$ for stage III disease. EP extraperigastric, $P G$ perigastric

LNs in gastric cancer with submucosal invasion, and that the incidence of LN metastasis increased from 19.3 to $29.8 \%$. Furthermore, many studies [28-31] have reported the presence of micrometastasis in gastric cancer, although the clinical significance of micrometastasis that could not be detected by standard hematoxylin and eosin staining has yet to be determined. The missed metastatic and micrometastatic LNs could lead to an underestimation of the number of metastatic LNs in the skip group, and consequently the LN status would be understaged.

Another interesting finding of our study was that the number of LNs retrieved in the skip group was low, especially in the PG area. This is important, as the quality of surgery could affect the number of LNs retrieved. However, this number was high enough (over 40) to demonstrate a satisfactory LN dissection in all three groups, and the quality of surgery may affect the number of LNs retrieved from the EP area rather than from the PG area. Two hypotheses would explain this finding: (1) less developed LNs around the PG area cause the bypass or direct lymphatic flow to the EP area in the skip group, or (2) inadequate examination of LNs results in those responsible for finding the LNs after surgery for pathologic confirmation neglecting or missing some LNs in the PG area. When the two findings (poor prognosis of the skip group and fewer LNs retrieved around the PG area in the skip group) are considered together, skip metastasis seems to be caused by inadequate examination of LNs. However, it is difficult to conclude whether skip metastasis is really the skipping of cancer cells which bypass the lymphatic 

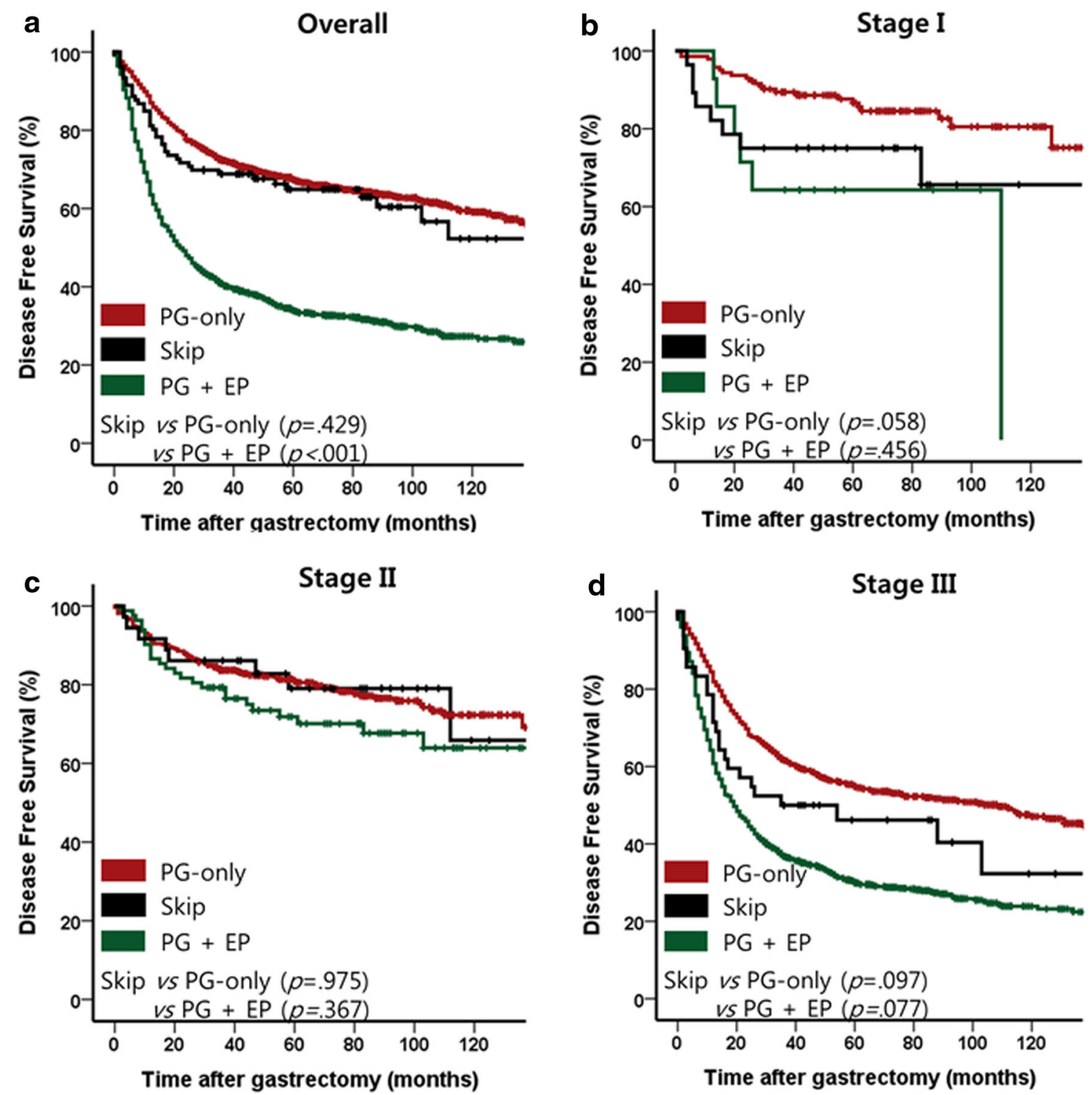

Fig. 2 Disease-free survival of each status of lymph node metastasis with Kaplan-Meier curves before matching: a for overall stage, $\mathbf{b}$ for stage I disease, $\mathbf{c}$ for stage II disease, and $\mathbf{d}$ for stage III disease. $E P$ extraperigastric, $P G$ perigastric

network around the PG area or if metastatic and micrometastatic LNs were skipped by inadequate examination, as there is no way to know the causal relationship of each with this retrospective study. Thus, we intend to conduct further studies investigating the LNs in the PG area of the skip group to discover clues as to the mechanism of skip metastasis.

The incidence of skip metastasis in previous reports was heterogeneous, and the different populations in each study seemed to be the main causative factor. In our study, the incidence of skip metastasis was $1.0 \%$ (31 of 3,010) for early gastric cancer, $10.7 \%$ (31 of 289) for early gastric cancer with LN metastasis, $2.5 \%$ (75 of 3,015) for advanced gastric cancer, $3.9 \%$ (75 of 1,942) for advanced gastric cancer with LN metastasis, and $13.5 \%$ (60 of 444) in patients with a single metastatic $\mathrm{LN}$ regardless of $\mathrm{pT}$ stage. These findings are similar to those of previous reports $[8,17,24]$. In addition, most of those previous reports were focused on the possibility of sentinel LN navigation and early gastric cancer. Thus, the number of skip metastasis cases was very limited, and the prognosis of skip metastasis could not be evaluated. In our study, only $29.2 \%$ of cases (31 of 106) of skip metastasis were early gastric cancer. Further studies are needed to review the entire gastric cancer population in order to scrutinize the mechanism of lymphatic metastasis in gastric cancer, especially as it pertains to skip metastasis.

The extent of LN dissection in gastric cancer has long been a disputed issue; however, recently, most guidelines [31-33] recommend that D2 LN dissection should be 

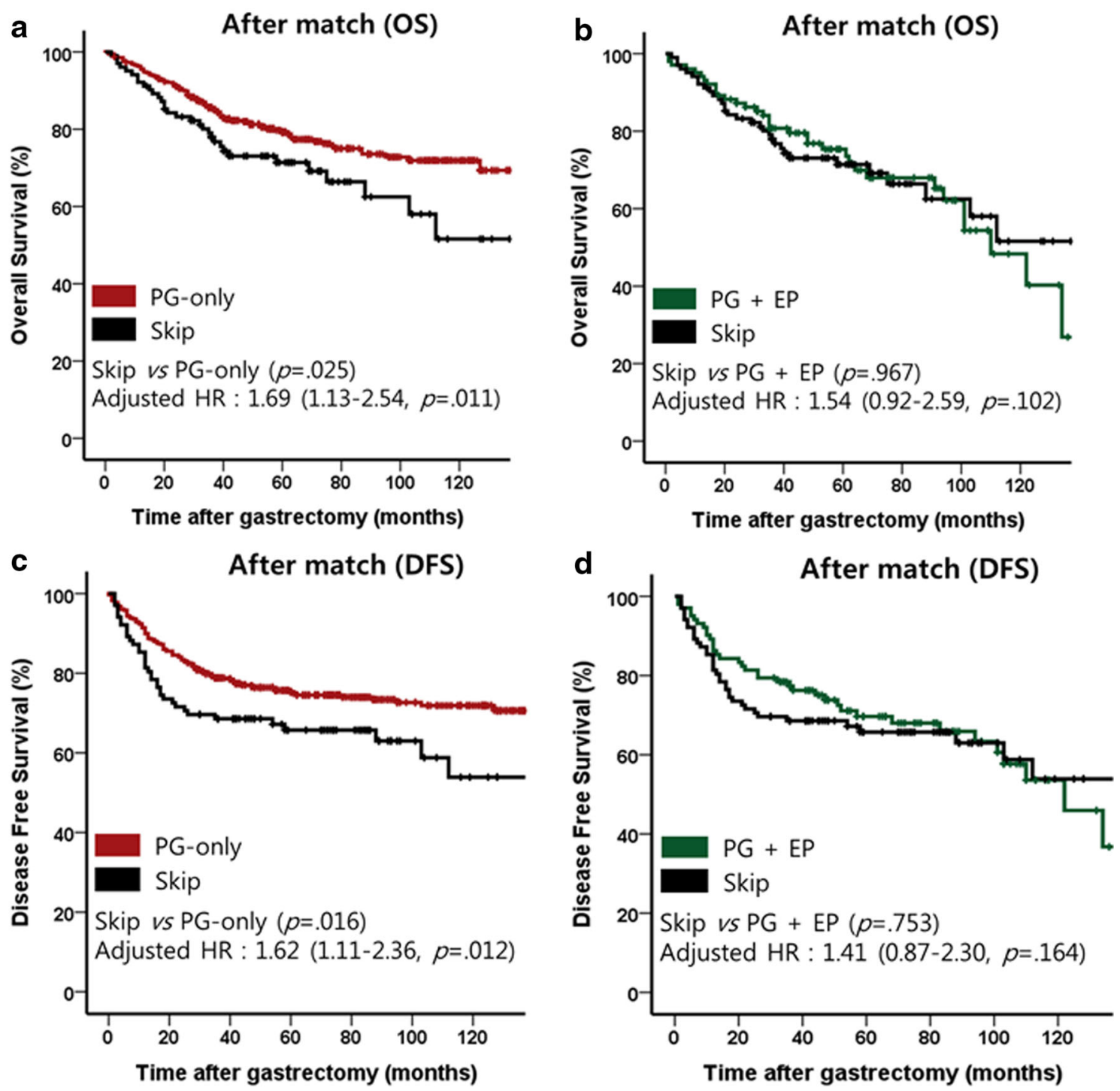

Fig. 3 Overall survival $(O S)$ and disease-free survival (DFS) of each status of lymph node metastasis with Kaplan-Meier curves: a OS of the skip group versus the perigastric $(P G)$-only group after matching, b OS of the skip group versus the $\mathrm{PG}+$ extraperigastric $(E P)$ group

after matching, c DFS of the skip group versus the PG-only group after matching, and $\mathbf{d}$ DFS of the skip group versus the PG + EP group after matching. $H R$ hazard ratio

performed, especially for advanced gastric cancer. Our results showed that most cases of skip metastasis were located at stations $7,8 \mathrm{a}$, and 9 , but that the incidence of skip metastasis at stations 10,11 , and 12 a was over $10 \%$. Also, the incidence of skip metastasis was $2.5 \%$ in advanced gastric cancer and $4.8 \%$ in LN-positive gastric cancer. If D2 gastrectomy were not performed in this case, the final pathologic stage could be underestimated, or even worse, R0 resection could not be achieved. Despite the improved diagnostic tools and technologies, to date there is no way of knowing the presence of skip metastasis before and even during surgery. Thus, D2 gastrectomy is recommended for advanced gastric cancer with $\mathrm{LN}$ involvement if it can be performed safely.

As with most retrospective studies, selection bias is a possible limitation of our study. Along these lines, excluding part of the population because the location of the LNs was not divided or the location of metastatic LNs was unknown could increase the selection bias. To minimize the selection bias for comparing the prognosis of the skip group with that of the other groups, we applied several statistical methods, such as propensity score match and adjustment. Additionally, the possibility of inaccurate classification and contamination of LN stations is another limitation. However, the information for defining skip metastasis is less ambiguous, as classifying the PG area and the EP area is clearer than defining the exact location of each LN. 


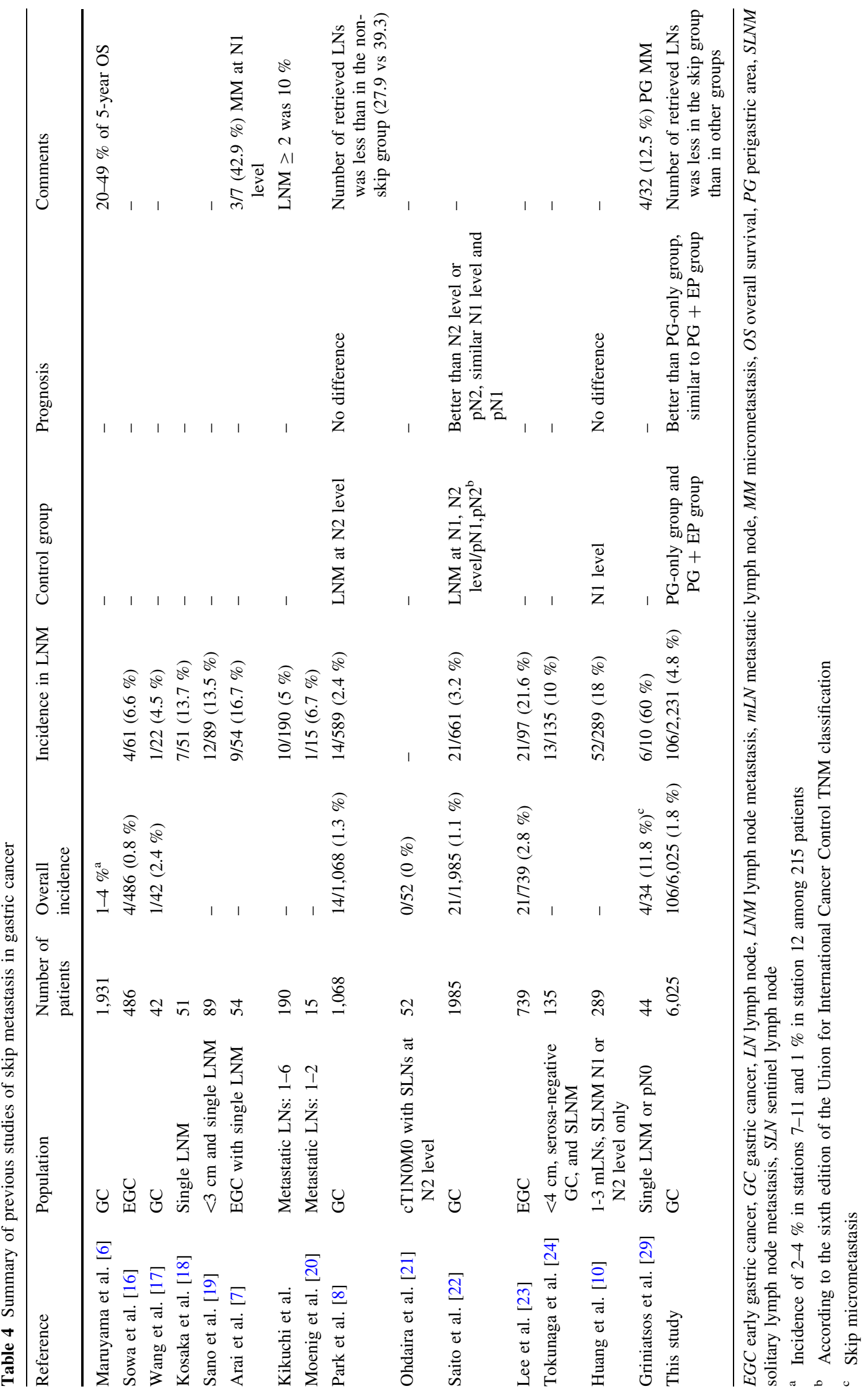


In conclusion, the prognosis of the skip group was worse than that of the PG-only group and similar to that of the PG + EP group when the tumor stage was considered. In addition, we found that the number of LNs retrieved was less in the skip group than in the other groups, especially in the PG area. However, whether it is the skipping of cancer cells or their having been skipped by inadequate examination of LNs, the mechanism of skip metastasis is still uncertain. More evidence should be accumulated for this unusual pattern of LN metastasis in gastric cancer to better define its mechanism.

Acknowledgments This study was supported by a grant from the National R\&D Program for Cancer Control, Ministry of Health and Welfare, Republic of Korea $(1320360,1020390)$. The authors are grateful to Dong-Su Jang, (Medical Illustrator, Medical Research Support Section, Yonsei University College of Medicine, Seoul, Korea) for his help with the illustrations.

Conflict of interest The authors declare that they have no conflict of interest or financial ties.

\section{References}

1. Kim JP, Kim YW, Yang HK, Noh DY. Significant prognostic factors by multivariate analysis of 3926 gastric cancer patients. World J Surg. 1994;18:872-7; discussion 877-8.

2. Siewert JR, Bottcher K, Stein HJ, Roder JD. Relevant prognostic factors in gastric cancer: ten-year results of the German Gastric Cancer Study. Ann Surg. 1998;228:449-61.

3. Sun J, Yin J, Ning L, Liu J, Liu H, Gu L, et al. Clinicopathological characteristics of breast cancers with axillary skip metastases. J Invest Surg. 2012;25:33-6.

4. Shiozawa M, Akaike M, Yamada R, Godai T, Yamamoto N, Saito $\mathrm{H}$, et al. Clinicopathological features of skip metastasis in colorectal cancer. Hepatogastroenterology. 2007;54:81-4.

5. Komatsu H, Mizuguchi S, Izumi N, Chung K, Hanada S, Inoue H, et al. Sialyl Lewis $\mathrm{X}$ as a predictor of skip N2 metastasis in clinical stage IA non-small cell lung cancer. World J Surg Oncol. 2013;11:309.

6. Maruyama K, Gunven P, Okabayashi K, Sasako M, Kinoshita T. Lymph node metastases of gastric cancer. General pattern in 1931 patients. Ann Surg. 1989;210:596-602.

7. Arai K, Iwasaki Y, Takahashi T. Clinicopathological analysis of early gastric cancer with solitary lymph node metastasis. Br J Surg. 2002;89:1435-7.

8. Park SS, Ryu JS, Min BW, Kim WB, Kim SJ, Kim CS, et al. Impact of skip metastasis in gastric cancer. ANZ J Surg. 2005;75:645-9.

9. Li C, Kim S, Lai JF, Oh SJ, Hyung WJ, Choi WH, et al. Solitary lymph node metastasis in gastric cancer. J Gastrointest Surg. 2008;12:550-4.

10. Huang B, Wang Z, Sun Z, Zhao B, Xu H. A novel insight of sentinel lymph node concept based on 1-3 positive nodes in patients with pT1-2 gastric cancer. BMC Cancer. 2011;11:18.

11. Miwa K, Kinami S, Taniguchi K, Fushida S, Fujimura T, Nonomura A. Mapping sentinel nodes in patients with early-stage gastric carcinoma. Br J Surg. 2003;90:178-82.

12. Maruyama K, Gunven P, Okabayashi K, Sasako M, Kinoshita T. Lymph node metastases of gastric cancer. General pattern in 1931 patients. Ann Surg. 1989;210:596-602.
13. Gervasoni JE Jr, Taneja C, Chung MA, Cady B. Biologic and clinical significance of lymphadenectomy. Surg Clin North Am. 2000;80:1631-73.

14. Japanese Gastric Cancer A. Japanese classification of gastric carcinoma: 3rd English edition. Gastric Cancer. 2011;14:101-12.

15. Rubin DB. Estimating causal effects from large data sets using propensity scores. Ann Intern Med. 1997;127:757-63.

16. Sowa M, Kato Y, Nishimura M, Kubo T, Maekawa H, Umeyama K. Surgical approach to early gastric cancer with lymph node metastasis. World J Surg. 1989;13:630-5 Discussion 5-6.

17. Wang LS, Wu CW, Hsieh MJ, Fahn HJ, Huang MH, Chien KY. Lymph node metastasis in patients with adenocarcinoma of gastric cardia. Cancer. 1993;71:1948-53.

18. Kosaka T, Ueshige N, Sugaya J, Nakano Y, Akiyama T, Tomita $\mathrm{F}$, et al. Lymphatic routes of the stomach demonstrated by gastric carcinomas with solitary lymph node metastasis. Surg Today. 1999;29:695-700.

19. Sano T, Katai H, Sasako M, Maruyama K. Gastric lymphography and detection of sentinel nodes. Recent Results Cancer Res. 2000;157:253-8.

20. Moenig SP, Luebke T, Baldus SE, Schroeder W, Bollschweiler E, Schneider PM, et al. Feasibility of sentinel node concept in gastric carcinoma: clinicopathological analysis of gastric cancer with solitary lymph node metastases. Anticancer Res. 2005;25:1349-52.

21. Ohdaira H, Nimura H, Mitsumori N, Takahashi N, Kashiwagi H, Yanaga K. Validity of modified gastrectomy combined with sentinel node navigation surgery for early gastric cancer. Gastric Cancer. 2007;10:117-22.

22. Saito H, Tsujitani S, Ikeguchi M. Clinical significance of skip metastasis in patients with gastric cancer. Gastric Cancer. 2007;10:87-91.

23. Lee SE, Lee JH, Ryu KW, Cho SJ, Lee JY, Kim CG, et al. Sentinel node mapping and skip metastases in patients with early gastric cancer. Ann Surg Oncol. 2009;16:603-8.

24. Tokunaga M, Ohyama S, Hiki N, Fukunaga T, Yamada K, Sano $\mathrm{T}$, et al. Investigation of the lymphatic stream of the stomach in gastric cancer with solitary lymph node metastasis. World J Surg. 2009;33:1235-9.

25. Son T, Hyung WJ, Kim JW, Kim HI, An JY, Cheong JH, et al. Anatomic extent of metastatic lymph nodes: still important for gastric cancer prognosis. Ann Surg Oncol. 2013;21(3):899-907.

26. Natsugoe S, Aikou T, Shimada M, Yoshinaka H, Takao S, Shimazu $\mathrm{H}$, et al. Occult lymph node metastasis in gastric cancer with submucosal invasion. Surg Today. 1994;24:870-5.

27. Aikou T, Higashi H, Natsugoe S, Hokita S, Baba M, Tako S. Can sentinel node navigation surgery reduce the extent of lymph node dissection in gastric cancer? Ann Surg Oncol. 2001;8:90-3.

28. Cai J, Ikeguchi M, Maeta M, Kaibara N, Sakatani T. Clinicopathological value of immunohistochemical detection of occult involvement in pT3N0 gastric cancer. Gastric Cancer. 1999;2:95-100.

29. Griniatsos J, Yiannakopoulou E, Gakiopoulou H, Alexandrou A, Dimitriou N, Karavokyros I, et al. Clinical implications of the histologically and immunohistochemically detected solitary lymph node metastases in gastric cancer. Scandinavian J Surg. 2011;100:174-80.

30. Doekhie FS, Mesker WE, van Krieken JH, Kok NF, Hartgrink $\mathrm{HH}$, Kranenbarg EK, et al. Clinical relevance of occult tumor cells in lymph nodes from gastric cancer patients. Am J Surg Pathol. 2005;29:1135-44.

31. Ajani JA, Bentrem DJ, Besh S, D'Amico TA, Das P, Denlinger C, et al. Gastric cancer, version 2.2013: featured updates to the NCCN guidelines. J Natl Compr Canc Netw. 2013;11:531-46.

32. Okines A, Verheij M, Allum W, Cunningham D, Cervantes A, Group EGW. Gastric cancer: ESMO clinical practice guidelines for diagnosis, treatment and follow-up. Ann Oncol. 2010;21(Suppl 5):v50-4.

33. Japanese Gastric Cancer Association. Japanese gastric cancer treatment guidelines 2010 (ver. 3). Gastric Cancer. 2011;14:113-23. 